\title{
Prevention of Hyperuricemia by Clerodendrum trichotomum Leaf Extract in Potassium Oxonate-Induced Mice
}

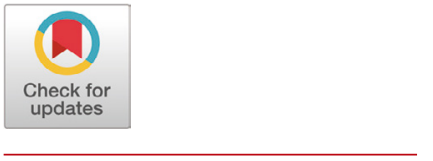

Received: May 4, 2020

Revised: May 11, 2020

Accepted: May 20, 2020

${ }^{\dagger}$ Corresponding author

Se-Jae Kim

Dept. of Biology, Jeju National University, Jeju 63243, Korea.

Tel: +82-64-754-3529

Fax: +82-64-726-3539

E-mail: sjkim@jejunu.ac.kr

Copyright (c) 2020 The Korean Society of Developmental Biology.

This is an Open Access article distributed under the terms of the Creative Commons Attribution Non-Commercial License (http://creativecommons.org/licenses/ by-nc/4.0/) which permits unrestricted non-commercial use, distribution, and reproduction in any medium, provided the original work is properly cited.

ORCID

Mi Gyeong Jang

https://orcid.org/0000-0002-4489-1365

Hana Song

https://orcid.org/0000-0002-2857-2110 Ji Hye Kim

https://orcid.org/0000-0003-0520-4478 Jung Min Oh

https://orcid.org/0000-0003-1567-8132 Jung Young Park

https://orcid.org/0000-0001-5728-4305 Hee Chul Ko

https://orcid.org/0000-0002-0222-6658

Sung-Pyo Hur

https://orcid.org/0000-0002-1622-3271

Se-Jae Kim

https://orcid.org/0000-0001-9058-326X

Conflict of interests

The authors declare no potential conflict of interest.

\author{
Mi Gyeong Jang ', Hana Song ${ }^{1}$, Ji Hye Kim¹, Jung Min Oh 1, Jung Young Park', \\ Hee Chul Ko ${ }^{2}$, Sung-Pyo Hur ${ }^{3}$, and ${ }^{\dagger}$ Se-Jae Kim ${ }^{1,2}$ \\ 'Dept. of Biology, Jeju National University, Jeju 63243, Korea \\ ${ }^{2}$ Biotech Regional Innovation Center, Jeju National University, Jeju 63243, Korea \\ ${ }^{3}$ Korea Institute of Ocean Science \& Technology, Jeju 63243, Korea
}

\begin{abstract}
Clerodendrum trichotomum is a folk medicine exhibiting anti-hypertension, anti-arthritis, and anti-rheumatism properties. However, little is known about whether the material might prevent hyperuricemia and associated inflammation. In this study, we explored whether $C$. trichotomum leaf extract (CTE) prevented hyperuricemia induced by potassium oxonate (PO) in mice. CTE (400 mg/kg body weight) significantly reduced the serum uric acid (UA), blood urea nitrogen (BUN), and serum creatinine levels and increased urine UA and creatinine levels. CTE ameliorated PO-induced inflammation and apoptosis by reducing the levels of relevant proteins in kidney tissues. Also, CTE ameliorated both UA-induced inflammatory response in RAW 263.7 cells and UA-induced cytotoxicity in HK-2 cells. In addition, liver transcriptome analysis showed that CTE enriched mainly the genes for mediating positive regulation of MAPK cascade and apoptotic signaling pathways. Together, the results show that CTE effectively prevents hyperuricemia and associated inflammation in PO-induced mice.
\end{abstract}

Keywords: Clerodendrum trichotomum, Hyperuricemia, Potassium oxonate, Transcriptome, Uric acid

\section{INTRODUCTION}

Uric acid (UA) is the terminal product of purine metabolism and is produced from hypoxanthine by xanthine oxidase (XO) of the liver (Wu et al., 1992). The serum UA level is determined by the extent of de novo purine synthesis, tissue catabolism, and exogenous proteins delivered to the liver (Kikuchi et al., 2017). UA excretion is controlled by kidney transporters, renal plasma flow, glomerular filtration, and proximal tubular exchange (George \& Struthers, 2009; So \& Thorens, 2010). An imbalance between UA production and excretion induces hyperuricemia, a major cause of gout, obesity, cardiovascular and renal diseases, hypertension, and metabolic syndrome (Edwards, 2009). Though UA exerted the modest antioxidant activity, hyperuricemia is a potentially harmful condition. It favors deposition of UA 
Acknowledgements

This research was supported by Basic Science

Research Program through the National

Research Foundation of Korea (NRF) by the

Ministry of Education, Science and Technology

(2017R1D1A3B03029845), Korea.

\section{Authors' contributions}

Conceptualization: Kim SJ.

Data curation: Ko HC.

Formal analysis: Hur SP.

Methodology: Song $\mathrm{H}$.

Software: Park JY.

Validation: Kim JH.

Investigation: Oh JM.

Writing - original draft: Jang MG.

Writing - review \& editing: Kim SJ.

\section{Ethics approval}

All experiments were approved by the IACUC of Jeju National University (approval no. 20160043). crystals in the joints and kidneys (Grassi et al., 2013). UA generates oxidative stress in kidney cells, promotes apoptosis by inducing imbalances of anti-apoptosis proteins and pro-apoptosis proteins, and causes inflammatory and endothelial dysfunction (Quan et al., 2011; Verzola et al., 2014).

Allopurinol (AP; an inhibitor of XO) is the synthetic drug most widely used to treat hyperuricemia (Pacher et al., 2006); its use is associated with certain side effects such as gastrointestinal distress, hypersensitivity reactions, rash, eosinophilia, and worsening of renal function (Pacher et al., 2006; Feig et al., 2008). Hence, searching for new agents with fewer side effects are required. Natural products (complex bioactive compounds) are valuable sources of novel potent pharmaceuticals with few side effects. Many studies have examined the use of natural products to treat hyperuricemia (Zhu et al., 2004).

Clerodendrum trichotomum Thunb of the Verbenaceae grows in fields and mountains of Korea, Japan, and China (Lee, 1973). The leaves and stems that used to treat arthritis and hypertension (Kim et al., 2009), exhibit diverse pharmacological activities (antihypertensive, sedative, analgesic, and anti-inflammatory properties; Ahn, 2003; Neeta and Tejas, 2007). Phenylethanoid and flavonoid glycosides and abietane diterpenoids have been isolated and characterized from the leaves and stems (Wang et al., 2013). However, their possible anti-hyperuricemic effects have not yet studied. Here, we explored the hypouricemic potential of $C$. trichotomum leaf extract (CTE) in potassium oxonate (PO)-induced mice. In addition, we investigated the transcriptome changes by CTE administration in livers of PO-induced mice using DNA microarray analysis.

\section{MATERIALS AND METHODS}

\section{Plant material and extraction}

C. trichotomum leaves were collected from the northern part of Jeju Island in July 2016. The leaves were washed, dried, and pulverized to powder of 100-200 mesh. The powders were extracted for $4 \mathrm{~h}$ with hot water $\left(90^{\circ} \mathrm{C}\right)$. CTE was concentrated, freeze-dried, and stored at $-70^{\circ} \mathrm{C}$ until use.

\section{Cell culture}

Human renal tubular epithelial HK-2 cells and murine macrophage RAW 264.7 cells were purchased from the Korean Cell Line Bank (Seoul, Korea). Cells were cultured in Roswell Park Memorial Institute-1640 medium (RPMI-1640, Gibco, Grand Island, NY, USA) supplemented with $10 \%$ fetal bovine serum and 1\% penicillin-streptomycin (Gibco) or Dulbecco's modified Eagle's medium (DMEM, Gibco) containing 10\% fetal bovine serum and 1\% penicillinstreptomycin (Gibco) at $37^{\circ} \mathrm{C}$ in a $5 \% \mathrm{CO}_{2}$.

\section{Cell viability}

The cell viability was assessed using the MTT assay. HK-2 cells were seeded into 96-well plates at $2 \times 10^{5} \mathrm{cell} / \mathrm{mL}$ and allowed to adhere overnight. After cells were pre-treatment with various concentrations of CTE for $1 \mathrm{~h}$, UA ( $20 \mathrm{mg} / \mathrm{dL})$ was added and cultured for $48 \mathrm{~h}$. RAW 264.7 cells were pretreated with indicated concentration of CTE for $1 \mathrm{~h}$, and then co-treated with UA $(20 \mathrm{mg} / \mathrm{dL})$ and LPS $(100 \mathrm{ng} / \mathrm{mL})$ for $24 \mathrm{~h}$. After then, each well was supplemented with $50 \mu \mathrm{L}$ of MTT and incubated for $4 \mathrm{~h}$ at $37^{\circ} \mathrm{C}$. The formazan crystals formed were subsequently dissolved in $200 \mu \mathrm{L}$ DMSO, and the optical density of the resultant reaction solution was read at $595 \mathrm{~nm}$ using a microplate reader (Bio-Tek, Winooski, VT, USA). 


\section{Animals and drug administration}

Male ICR mice (5 weeks of age, $30 \pm 2 \mathrm{~g}$ in body weight [BW]) were purchased from Orient Bio (Seongnam, Korea). All animals were allowed free access to water and standard mouse chow and were held under a regular $12-\mathrm{h}$ light/dark cycle at $23 \pm 2{ }^{\circ} \mathrm{C}$ and relative humidity of $60 \pm 5 \%$. The animals were acclimatized to the environment for 7 days and then used in experiments. All experiments were approved by the Animal Care and Use Committee of Jeju National University (approval no. 2016-0043). The uricase inhibitor PO was used to induce hyperuricemia (Wang et al., 2015). PO (250 mg/kg) was intraperitoneally administered daily $1 \mathrm{~h}$ before drug administration for 7 consecutive days. The mice were randomly divided into four groups ( $\mathrm{n}=6$ per group). Normal control (NC) mice were fed only the basic diet. A PO-induced hyperuricemic group (PO+50 mg saline/kg BW), an AP group (PO+5 mg AP/kg of BW), and a CTE group (PO+400 mg CTE/kg $\mathrm{BW})$; the $\mathrm{AP}$ and $\mathrm{CTE}$ were given on the day after $\mathrm{PO}$ was given.

\section{Biochemical parameter assays}

After 6 days of drug administration, urine samples were collected for $24 \mathrm{~h}$ in a metabolic cage and centrifuged. The supernatants were collected, and used for UA and creatinine content assays. On the last day (day 7), all mice were anesthetized with ethyl ether. Blood samples were drawn from the heart into a syringe and allowed to stand at room temperature. Serum samples were then collected by centrifugation at $800 \times \mathrm{g}$ for $10 \mathrm{~min}$. The tissues were extracted, rapidly cooled in liquid nitrogen, and stored at $-70^{\circ} \mathrm{C}$. The serum and urine UA levels, blood urea nitrogen (BUN), and serum creatinine levels were measured using a UA kit (Abnova, Taipei, Taiwan), a BUN assay kit (Asan Pharm, Gyeonggi-do, Korea), and a creatinine assay kit (BioAssay Systems, Hayward, CA, USA) according to the manufacturers' protocols.

\section{Western blotting and histochemical analysis}

Cells and tissues were resuspended in cold lysis buffer $(1 \times$ RIPA buffer, $1 \mathrm{mM}$ phenylmethylsulfonyl fluoride, $1 \mathrm{mM} \mathrm{Na} \mathrm{VO}_{4}, 1 \mathrm{mM} \mathrm{NaF}, 1 \mu \mathrm{g} / \mathrm{mL}$ aprotinin, $1 \mu \mathrm{g} / \mathrm{mL}$ pepstatin, and $1 \mu \mathrm{g} / \mathrm{mL}$ leupeptin) and collected by centrifugation at $21,000 \times \mathrm{g}$ for $20 \mathrm{~min}$ at $4^{\circ} \mathrm{C}$. Lysate protein concentrations were determined using a protein assay featuring a dye reagent (BioRad; Hercules, CA, USA). The proteins were subjected to electrophoresis on 10\% (w/v) SDSpolyacrylamide gels and transferred to polyvinylidene fluoride membranes; the membranes were blocked with 5\% (w/v) skim milk and $0.1 \%(\mathrm{v} / \mathrm{v})$ Tween-20 in Tris-buffered saline for $1 \mathrm{~h}$ and then incubated with primary antibodies overnight at $4{ }^{\circ} \mathrm{C}$. The primary antibodies recognized the following proteins: Bax (Santa Cruz Biochemicals; Santa Cruz, CA, USA); Bcl-2 (Santa Cruz); caspase-3 (Santa Cruz); cleaved caspase-3 (Cell Signaling, Danvers, MA, USA); iNOS and TNF$\alpha$ (Santa Cruz); and COX-2 (BD Biosciences; Franklin Lakes, NJ, USA). The membranes were incubated at room temperature for $1 \mathrm{~h}$ with a peroxidase-conjugated secondary antibody (Vector Laboratories, Burlingame, CA, USA), and proteins were detected using the Westar ETA C 2.0 substrate (Cyanagen; Bologna, Italy). For histochemical analysis, liver tissues were fixed with paraformaldehyde, washed, dehydrated and embedded in paraffin. Then, a paraffin block was prepared and sectioned with a microtome to prepare a tissue slice. The serial paraffin sections were stained with hematoxylin and eosin solution. Immunostaining for tumor necrosis factor$\alpha$ (TNF- $\alpha$ ) was performed using TNF- $\alpha$ antibody (Life technologies, Carlsbad, CA, USA) and biotinylated goat-anti-rabbit IgG (Vector, USA). The histological changes were observed with a microscope (BX-51, Olympus, Tokyo, Japan). 


\section{DNA microarray and data analysis}

Total RNAs from liver tissues were isolated using Trizol reagent (Invitrogen, Carlsbad, CA, USA). To reduce variation among individuals within each experimental group, equal amounts of total RNA from individual within the same group were pooled together. RNA quality was assessed using the Agilent 2100 bioanalyzer and the RNA 6000 Nano Chip (Agilent Technologies, Amstelveen, The Netherlands), and RNA was quantified using the ND-2000 Spectrophotometer (Thermo Fisher Scientific, Waltham, MA, USA). DNA microarray was performed using Agilent Mouse GE 4 X 44K (V2). Briefly, amplification, labeling, hybridization, and wash were performed with Agilent's Low RNA Input Linear Amplification kit PLUS and Gene Expression Hybridization Kit, according to the manufacturer's instructions. The hybridization images were scanned by DNA microarray Scanner and the data analysis was performed using Agilent Feature Extraction software 10.7 and GeneSpringGX 7.3.1 (Agilent Technologies, Amstelveen, The Netherlands). Gene classification was based on searches of DAVID (http://david.abcc.ncifcrf.gov/) and Medline databases (http://www.ncbi.nlm.nih.gov/).

\section{Statistical analysis}

All statistical analyses were performed using SPSS ver. 12.0 for Windows (SPSS; Chicago, IL, USA). All data are shown as means \pm SEs. The differences between groups were examined via oneway ANOVA. A $p$-value $<0.05$ was considered to reflect statistical significance.

\section{RESULTS}

\section{Effects of CTE on uric acid, BUN, and creatinine levels}

The hypouricemic potential of CTE was assessed by measuring serum and urine UA levels. These UA levels were significantly increased in the PO group compared to the NC group (Fig. 1A), indicating that hyperuricemia was indeed induced by PO. The serum UA level in the CTE group was higher than that in the AP group (positive control), but lower than that in the $\mathrm{PO}$ group. However, the urine UA levels were higher in the CTE and AP groups than in the $\mathrm{PO}$ group (Fig. 1B). To evaluate whether $\mathrm{CTE}$ affected kidney function in mice with $\mathrm{PO}-$ induced mice, we measured the levels of creatinine and BUN in serum and urine. The serum creatinine level was significantly higher in the PO than in the CTE group (Fig. 1C). However, the urine creatinine level was significantly higher in the CTE than in the PO group (Fig. 1D). The serum BUN level in the CTE group was significantly lower than that in the PO group, being similar to those in the $\mathrm{NC}$ and $\mathrm{AP}$ groups (Fig. 1E).

\section{Effects of CTE on expression of inflammatory and cytoprotective proteins}

To explore whether CTE improve kidney function of PO-induced mice, the effects of CTE on the levels of inflammation- and apoptosis-related protein expressions were investigated via Western blotting. The levels of the inflammation-related proteins iNOS, COX-2, and TNF- $\alpha$ in kidney tissues were significantly higher in PO group than those of the $\mathrm{NC}$ group. However, the levels in the CTE group were lower than those in the $\mathrm{PO}$ group (Fig. 2A). Similarly, the levels of apoptosis-related proteins were affected by CTE treatment (Fig. 2B). The anti-apoptotic protein Bcl-2 was upregulated, and the pro-apoptotic protein Bax downregulated in both CTE and AP groups compared to the PO group. CTE increased the procaspase- 3 level and decreased that of cleaved caspase-3. Also, the immunohistochemical 
A

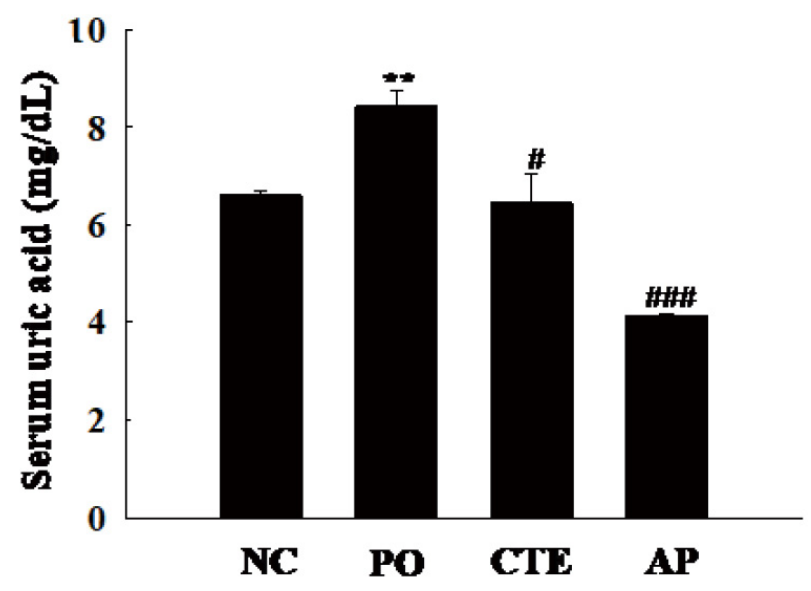

C

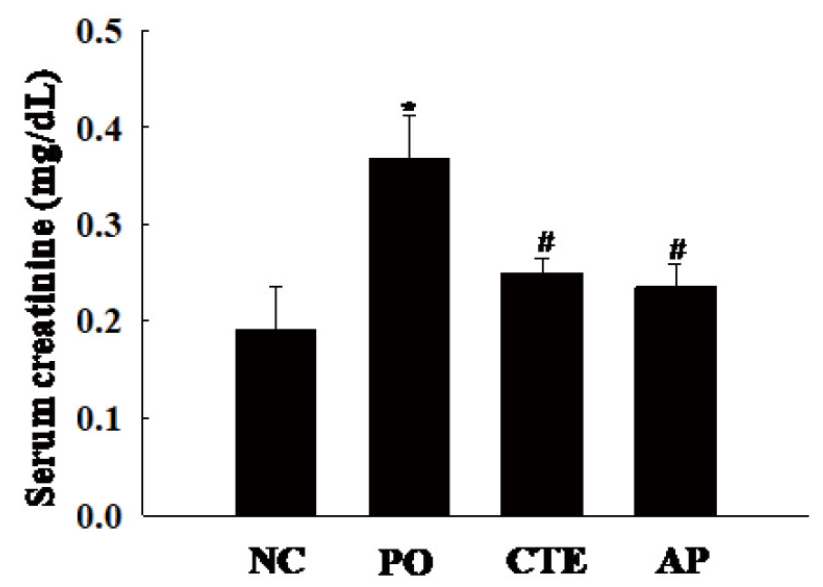

E

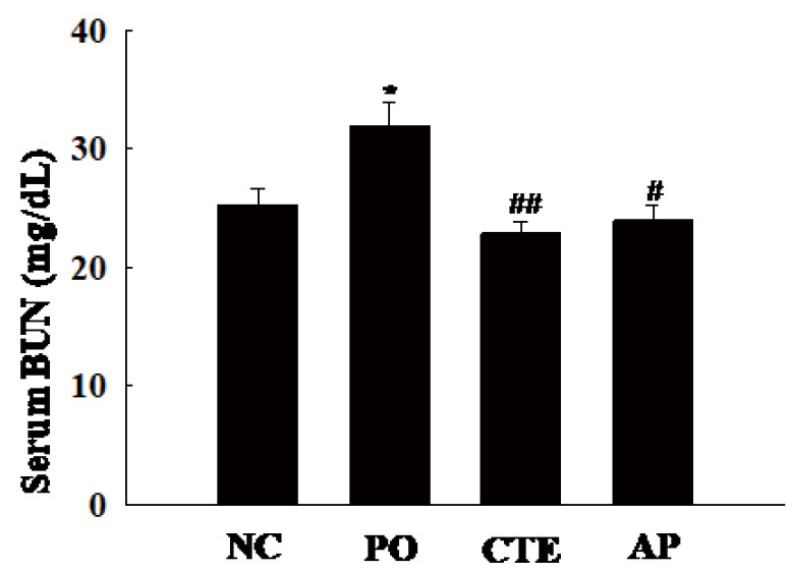

B

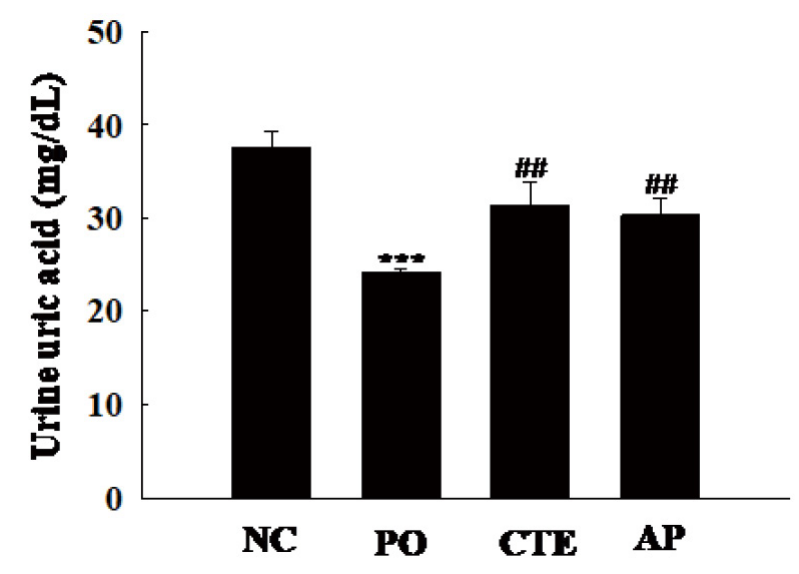

D

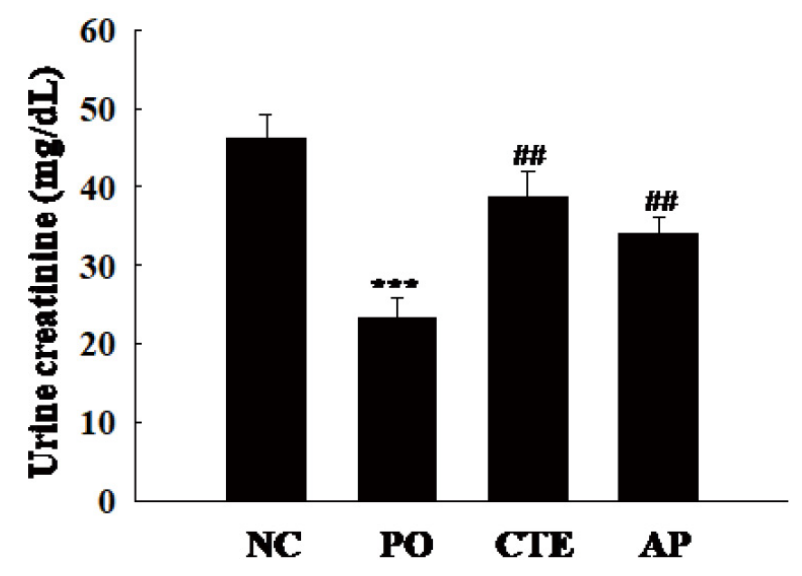

Fig. 1. Effects of CTE administration on parameters of impaired uric acid excretion and renal dysfunction in PO-induced mice. The levels of serum uric acid (A), urine uric acid (B), serum creatinine (C), urine creatinine (D), and serum BUN (E). NC, normal group; PO, potassium oxonate (250 mg/ $\mathrm{kg})$ group; CTE, PO+CTE $(400 \mathrm{mg} / \mathrm{kg})$ group; AP, PO+allopurinol $(5 \mathrm{mg} / \mathrm{kg})$ group. Each value is expressed as mean $\pm \mathrm{SE}(\mathrm{n}=3)$. " $p<0.05$, " $p<0.01 \mathrm{and}$ $p<0.001$ compared to normal group. ${ }^{\#} p<0.05,{ }^{\# \#} p<0.01$ and ${ }^{\# \#} p<0.001$ compared to PO group. CTE, Clerodendrum trichotomum leaf extract; BUN, blood urea nitrogen; AP, allopurinol. 
A

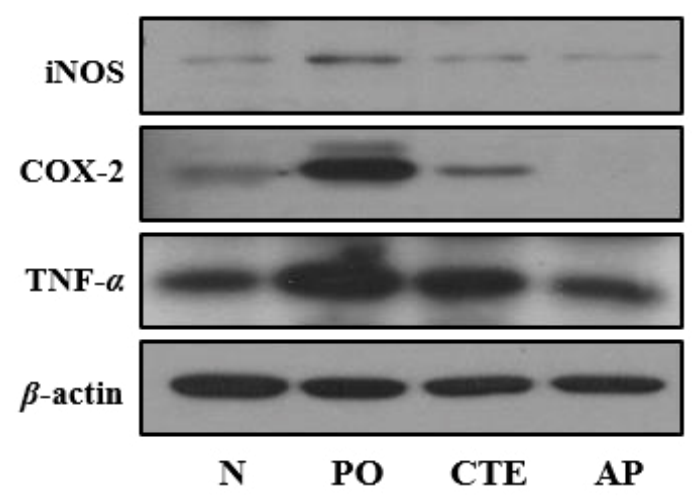

C
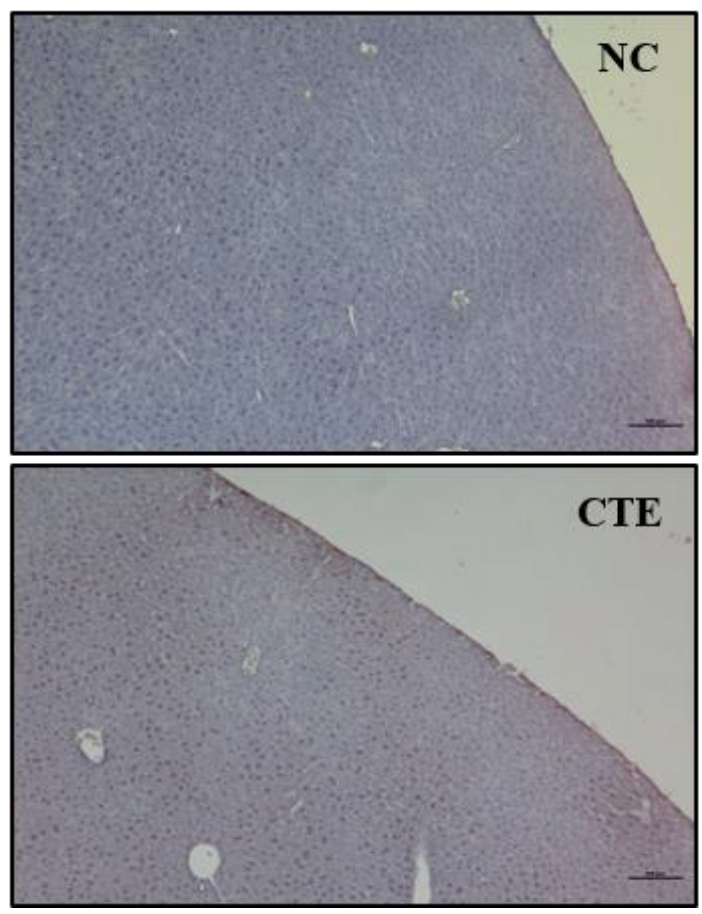

B
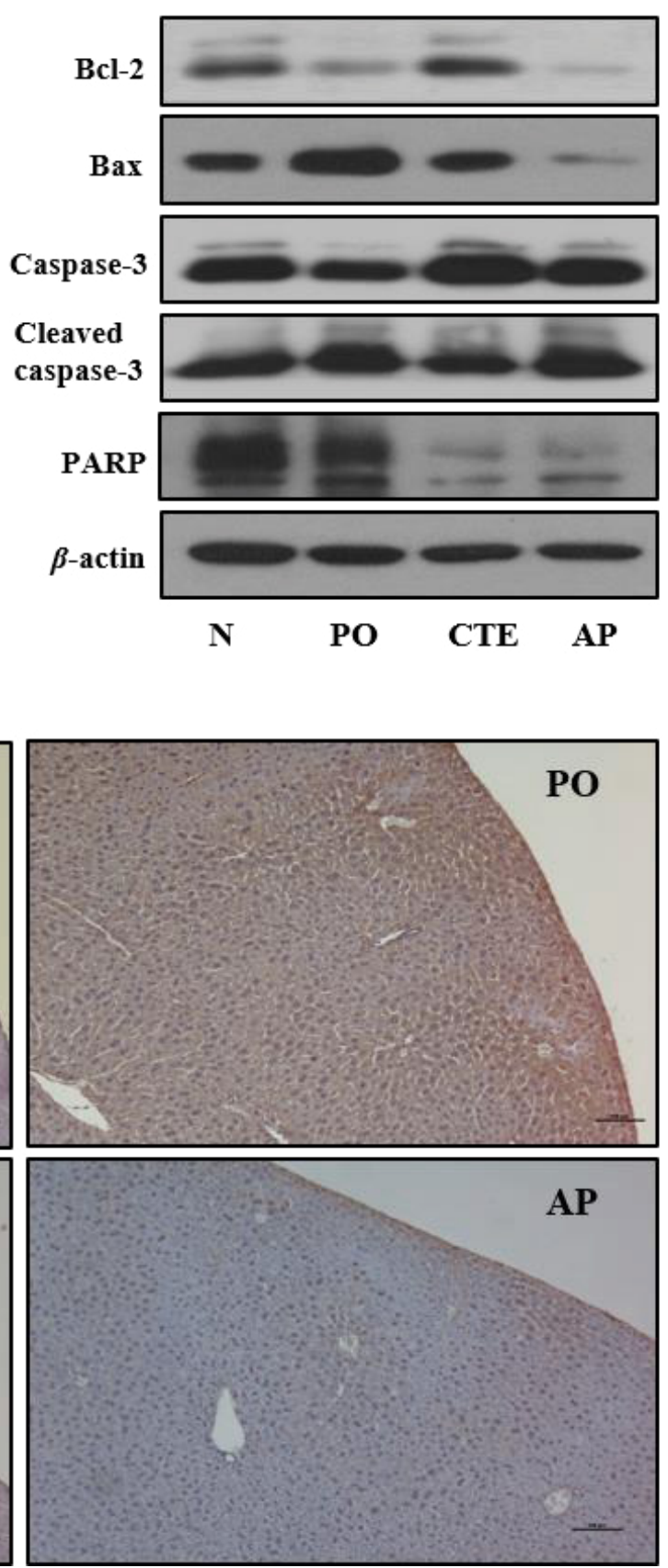

Fig. 2. Effects of CTE administration on expression of inflammatory and cytoprotective proteins in PO-induced mice. Western blots of inflammatory proteins (A), and apoptotic proteins (B) in the kidney tissues. Immunohistochemical staining TNF- $\alpha$ expression in livers tissues (magnification $\times 100$, scale

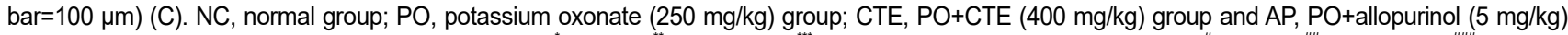
group. Each value is expressed as mean $\pm \mathrm{SE}(\mathrm{n}=3)$. ${ }^{*} p<0.05,{ }^{\prime *} p<0.01$ and ${ }^{* * * *} p<0.001$ compared to normal group. ${ }^{\#} p<0.05,{ }^{\#} p<0.01$ and ${ }^{\# \#} p<0.001$ compared to PO group. CTE, Clerodendrum trichotomum leaf extract; AP, allopurinol.

staining against TNF- $\alpha$ in liver tissues of PO-induced mice group showed clearly that the expression of TNF- $\alpha$ was higher in PO group compared to CTE and AP groups (Fig. 2C).

\section{Cytoprotective effects of CTE in uric acid-induced cells}

The effects of CTE on cell viability and expressions of cytoprotective protein in UA- 
induced cells were analyzed by MTT assay and Western blotting. As shown in Fig. 3A, CTE ameliorated dose-dependently the UA-induced cytotoxicity in HK-2 cells. Consistent with this result, CTE treatment decreased the expressions of proapoptotic protein Bax, and PARP and cleaved caspase-3, while it increased the expression of antiapoptotic protein Bcl2 in UA-induced HK-2 cells (Fig. 3B). Additionally, the anti-inflammatory activity of CTE was investigated in UA-induced RAW 264.7 cells. CTE treatment $(200 \mu \mathrm{g} / \mathrm{mL})$ decreased significantly UA-induced cytotoxicity in LPS-stimulated RAW 264.7 cells (Fig. 3C). LPS stimulated NO production in UA-induced RAW 264.7, but NO production was significantly reduced by CTE treatment (data not shown). Consistent with this result, CTE decreased the expression of pro-inflammatory proteins (iNOS and COX-2) and increased the expression of

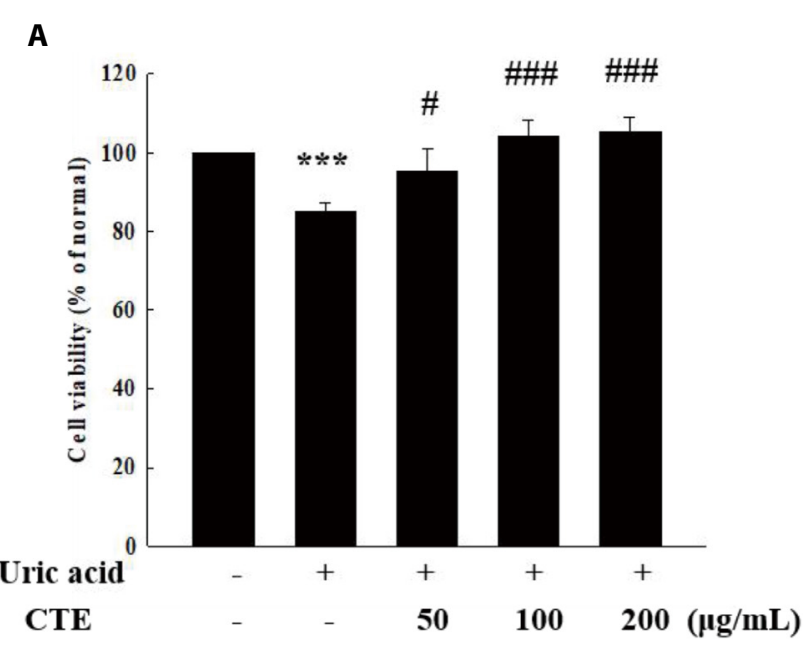

C

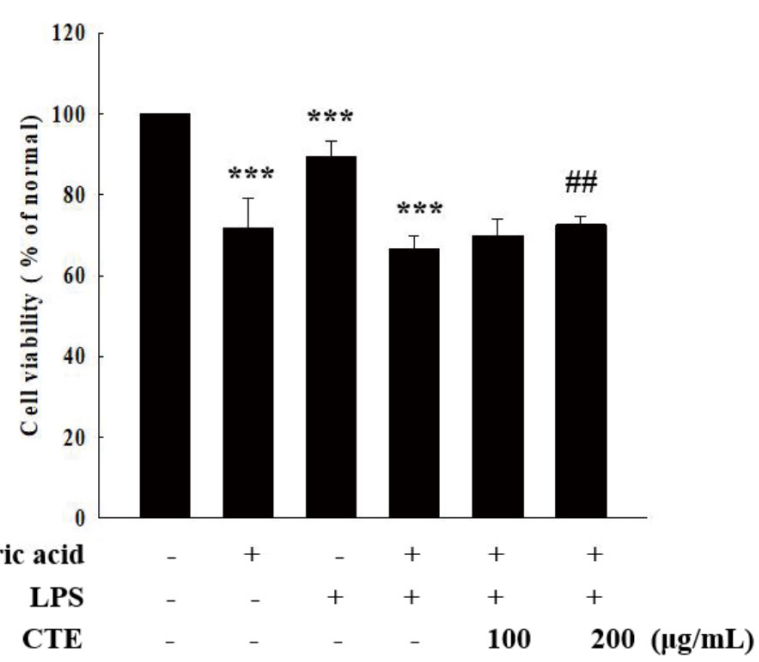

B

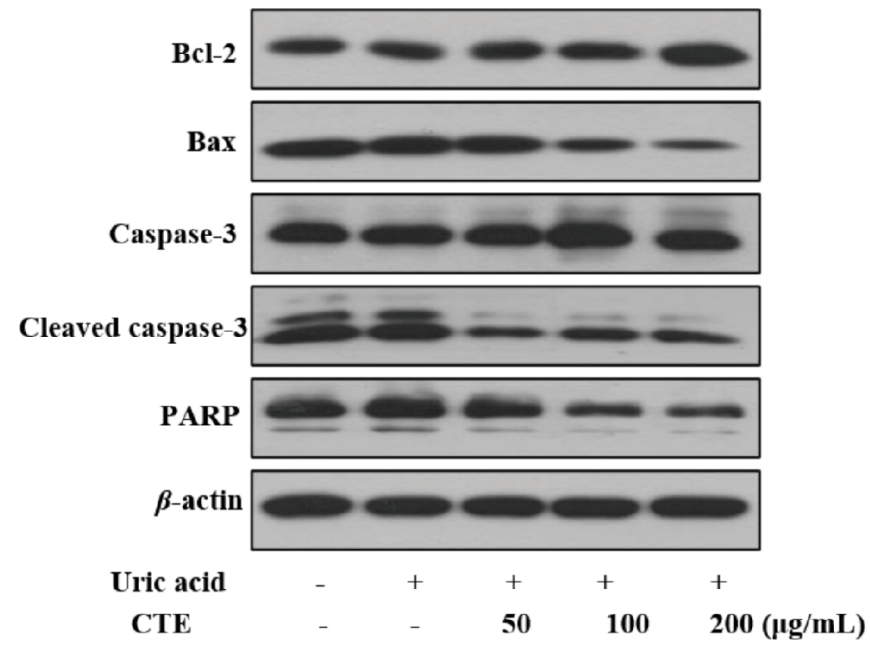

D

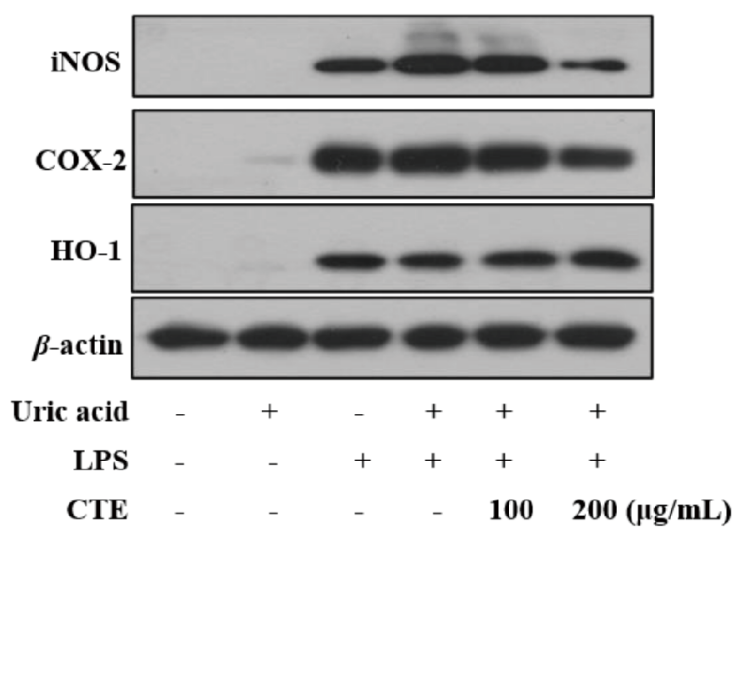

Fig. 3. Effects of CTE on cell viability, and expressions of apoptotic and inflammatory proteins in uric acid-induced HK-2 and RAW 274.7 cells. Cytotoxicity (A) and Western blot of apoptotic proteins (B) in HK-2 cells. Cytotoxicity (C) and Western blot of inflammatory proteins (D) in RAW264.7 cells. Each value is expressed as mean \pm SE $(n=3)$. ${ }^{*} p<0.05$ and ${ }^{* * *} p<0.001$, compared to non-treated group. ${ }^{\#} p<0.05$ and ${ }^{\# \#} p<0.001$ compared to uric acid-treated group. CTE, Clerodendrum trichotomum leaf extract. 
antioxidant protein, heme oxygenase-1 (HO-1) in UA-induced RAW 264.7 with LPS (Fig. 3D).

\section{Effect of CTE on liver transcriptome profiles in PO-induced mice}

To explore how CTE affects the global gene expressions in PO-induced mice, we analyzed the gene expression profiles of liver tissues via DNA microarray. Among 39,429 genes detected in microarray, we identified total 869 differentially expressed genes (DEGs) by comparing the transcriptome profiles in CTE versus PO groups (using 2.0-fold as the cut-off value). To understand the signatures of these DEGs, we performed Gene ontology (GO) and Kyoto Encyclopedia of Genes and Genomes (KEGG) pathway analysis. GO analysis revealed that $\mathrm{CTE}$ administration enriched significantly various $\mathrm{GO}$ biological processes, such as the extrinsic apoptotic signaling pathway in absence of ligand, positive regulation of MAPK cascade, negative regulation of endothelial cell apoptotic process, cholesterol metabolic process, apoptotic process, and cellular response to insulin stimulus $(p<0.05$, Table 1$)$. The pathway analysis showed that CTE administration enriched highly the pathways, such as Ras signaling, Rap1 signaling, glycerolipid metabolism, PPAR signaling, and VEGF signaling pathway $(p<0.05$, Table 2).

\section{DISCUSSION}

In recent years, morbidity due to hyperuricemia has rapidly increased in both young and elderly generations. Current hypuricemic agents, such as AP and benzbromarone, used for lowering serum UA are sometimes limited due to the associated unacceptable adverse events including fever, development of rashes, Steven-Johnson's syndrome, and AP hypersensitivity syndrome (Pacher et al., 2006; Feig et al., 2008). Therefore, there is a growing demand for edible hypouricemic ingredient that can complement the side effect of current therapeutic drugs. $C$. trichotomum is considered as a traditional anti-gout herbaceous plant due to its bioactivities, such as anti-hypertension and anti-inflammatory agent (Lee, 1973). The aim of this study was to investigate the hypouricemic potential of CTE in PO-induced mice.

It was well known that $\mathrm{PO}$ elevated the $\mathrm{XO}$ activity and UA level in mouse liver, and impaired renal function. CTE and AP administration significantly decreased the serum UA level, but increased urine UA level in PO-induced mice, although effect of CTE did not reached that of AP (positive control). Since it was reported that CTE had potent XO inhibitory activity (Kim et al., 2017), CTE presumably can reduce the production of UA in the liver. To evaluate the effect of CTE on kidney function in PO-induced mice, we measured the serum and urine creatinine and BUN levels. CTE administration decreased the serum creatinine level, but increased the urine creatinine level in PO-induced mice. The glomerular filtration rate is considered as an indicator of kidney function (Fan et al., 2014). Thus, it is suggested that CTE improve kidney function by controlling the BUN and creatinine levels. Together, these results suggest that CTE exert the hypouricemic activities by reducing UA production and increasing $\mathrm{UA}$ excretion in $\mathrm{PO}$-induced mice.

CTE administration decreased the expression of pro-inflammatory mediators (iNOS, $\mathrm{COX}-2$, and $\mathrm{TNF}-\alpha$ ) and pro-apoptotic protein (Bax), and increased anti-apoptotic protein (Bcl-2) in the kidney of PO-induced mice. Especially, the expression of pro-inflammatory cytokine, TNF- $\alpha$ was decreased by CTE administration in liver tissues of PO-induced mice. TNF- $\alpha$ is not expressed under normal conditions and increased in plasma or tissue when 
Table 1. Gene ontology (GO) analysis using differentially expressed genes (DEGs) observed in liver tissues of CTE versus PO groups

\begin{tabular}{|c|c|c|}
\hline Term & Count & $p$-value \\
\hline Cellular response to vascular endothelial growth factor stimulus & 7 & 4.15E-05 \\
\hline Positive regulation of angiogenesis & 14 & 9.51E-05 \\
\hline Sprouting angiogenesis & 7 & $2.20 \mathrm{E}-04$ \\
\hline Extrinsic apoptotic signaling pathway in absence of ligand & 8 & 2.74E-04 \\
\hline Transmembrane receptor protein tyrosine kinase signaling pathway & 11 & 0.001 \\
\hline Sterol biosynthetic process & 6 & 0.001 \\
\hline Positive regulation of MAPK cascade & 11 & 0.001 \\
\hline Negative regulation of endothelial cell apoptotic process & 6 & 0.002 \\
\hline Positive regulation of endothelial cell migration & 7 & 0.002 \\
\hline Cholesterol metabolic process & 10 & 0.002 \\
\hline Steroid metabolic process & 9 & 0.004 \\
\hline Neuron migration & 11 & 0.005 \\
\hline Neutrophil chemotaxis & 8 & 0.005 \\
\hline Oxidation-reduction process & 34 & 0.007 \\
\hline Angiogenesis & 16 & 0.008 \\
\hline T cell differentiation & 6 & 0.008 \\
\hline Cell surface receptor signaling pathway & 15 & 0.008 \\
\hline Lipid metabolic process & 25 & 0.009 \\
\hline Response to drug & 20 & 0.009 \\
\hline Receptor-mediated endocytosis & 7 & 0.010 \\
\hline Neural tube patterning & 3 & 0.013 \\
\hline Positive regulation of ERK1 and ERK2 cascade & 13 & 0.014 \\
\hline Protein oligomerization & 7 & 0.017 \\
\hline Apoptotic process & 28 & 0.019 \\
\hline Single organismal cell-cell adhesion & 9 & 0.019 \\
\hline Cellular response to insulin stimulus & 8 & 0.019 \\
\hline Positive regulation of cell migration & 13 & 0.024 \\
\hline Positive regulation of protein phosphorylation & 12 & 0.028 \\
\hline Positive regulation of cell adhesion & 6 & 0.029 \\
\hline Response to glucose & 7 & 0.034 \\
\hline Response to hypoxia & 12 & 0.037 \\
\hline Negative regulation of canonical Wnt signaling pathway & 8 & 0.039 \\
\hline Steroid biosynthetic process & 6 & 0.042 \\
\hline Potassium ion transport & 9 & 0.044 \\
\hline Blood coagulation & 7 & 0.046 \\
\hline Nervous system development & 19 & 0.047 \\
\hline Positive regulation of phosphatidylinositol 3-kinase signaling & 6 & 0.048 \\
\hline
\end{tabular}

The top 37 enriched GO term (biological processes) associated with the found DEGs. Count: number of annotated gens in the data. CTE, Clerodendrum trichotomum leaf extract; PO, potassium oxonate.

inflammation or infection occurs and plays an important role in immune, inflammation, differentiation, and apoptosis processes (Bradley, 2008; Shin et al., 2017). Especially, TNF- $\alpha$ induces an inflammatory response through the NF-кB pathway and the MAPK pathway, activates caspase-8, and induces apoptosis responses (Mohamed et al., 2002; Karin \& Gallagher 2009). Together, these results suggest that CTE prevent the tissue damages in PO- 
Table 2. KEGG pathway analysis using differentially expressed genes (DEGs) observed in liver tissues of CTE versus $\mathrm{PO}$ groups

\begin{tabular}{lcc}
\hline \hline \multicolumn{1}{c}{ KEGG pathway } & Count & $p$-value \\
\hline Ras signaling pathway & 16 & 0.008 \\
Rap1 signaling pathway & 15 & 0.011 \\
Glycerolipid metabolism & 7 & 0.012 \\
Circadian entrainment & 9 & 0.015 \\
PPAR signaling pathway & 8 & 0.016 \\
Complement and coagulation cascades & 7 & 0.038 \\
Transcriptional misregulation in cancer & 11 & 0.045 \\
VEGF signaling pathway & 6 & 0.047 \\
\hline
\end{tabular}

The top 8 significant pathways associated with the identified DEGs. Count: number of annotated genes in the data set. KEGG, kyoto encyclopedia of genes and genomes; CTE, Clerodendrum trichotomum leaf extract; PO, potassium oxonate.

induced mice via anti-inflammatory and cytoprotective activities.

In this study, we confirmed the effects of CTE in vitro. We analyzed the effects of CTE on cell viability and expressions of cytoprotective and inflammatory protein in UAinduced HK-2 and RAW 264.7 cells. CTE significantly reduced UA-induced cytotoxicity in HK-2 cells. Consistent with this result, CTE treatment decreased the expressions of proapoptotic protein Bax, and PARP and cleaved caspase-3, while it increased the expression of antiapoptotic protein $\mathrm{Bcl}-2$ in UA-induced $\mathrm{HK}-2$ cells, indicating its cytoprotective activity in HK-2 cells. Bcl-2 family, Bcl-2, Bcl-XL, etc. inhibit apoptosis, and Bax, Bad, and Bim play a role in promoting apoptosis. When oxidative stress caused by external harmful factors causes the balance of the Bcl-2 family to decrease and Bax expression increases, apoptosis occurs due to caspase chain reaction ( $\mathrm{Li}$ et al., 2016). Similarly, CTE treatment decreased the expression of pro-inflammatory proteins (iNOS and COX-2), and increased the expression of antioxidant heme oxygenase $1(\mathrm{HO}-1)$ protein in UA-induced RAW 264.7, indicting its anti-inflammatory activity. HO- 1 breaks down the heme to make carbon monoxide (CO), iron, and biliverdin, and biliverdin is converted to bilirubin by $\mathrm{NAD}(\mathrm{P}) \mathrm{H}$ biliverdin reductase (Ryter et al., 2002). And these converted compound inhibit iNOS and COX-2 expression and $\mathrm{NO}$ production, and are known to have antiapoptotic properties, antioxidant and antiinflammatory properties in various tissues and cells (Choi et al., 2012).

To explore the molecular mechanism on the hypouricemic action of CTE, we identified 869 DEGs in the liver of CTE versus PO groups using DNA microarray analysis. GO analysis showed that CTE administration regulated the expression of genes, which were involved in various biological process, such as extrinsic apoptotic signaling pathway in absence of ligand, positive regulation of MAPK cascade, cholesterol metabolic process, apoptotic process, and cellular response to insulin stimulus. KEGG pathway analysis revealed that these genes were involved in Ras signaling, Rap1 signaling, glycerolipid metabolism, PPAR signaling and VEGF signaling pathway. Taken together, these results suggest that CTE exert hypouricemic effect in part by regulating the genes, which are involved in cell signal transmitting and cytoprotective processes, thereby reducing both inflammation and apoptosis in PO-induced mice. A limitation of this study is that transcriptome analyses were performed on pooled samples from only five mice in CTE and PO (negative control) groups. However, the transcriptome data and the identified genes will serve as valuable references for further research to elucidate the molecular mechanisms of hypouricemic effect of CTE in POinduced mice. 
In summary, CTE administration exhibited the hypouricemic effect in PO-induced mice. CTE ameliorated PO-induced inflammation and apoptosis by reducing the levels of relevant proteins in kidney tissues. Also, CTE ameliorated both UA-induced inflammatory response in RAW 263.7 cells and UA-induced cytotoxicity in HK-2 cells. In addition, CTE enriched mainly the genes for mediating positive regulation of MAPK cascade and apoptotic signaling pathways. These findings suggested that CTE might be promising dietary ingredients against hyperuricemia with inflammation.

\section{REFERENCES}

Ahn DK (2003) Illustrated Book of Korean Medicinal Herbs. 6th ed. Kyo-Hak, Seoul, Korea, p 323.

Bradley JR (2008) TNF-mediated inflammatory disease.J Pathol 214:149-160.

Choi HG, Lee DS, Li B, Choi YH, Lee SH, Kim YC (2012) Santamarin, a sesquiterpene lactone isolated from Saussurea lappa, represses LPS-induced inflammatory responses via expression of heme oxygenase-1 in murine macrophage cells. Int Immunopharmacol 13:271-279.

Edwards NL (2009) The role of hyperuricemia in vascular disorders. Curr Opin Rheumatol 21:132-137.

Fan CY, Wang MX, Ge CX, Wang X, Li JM, Kong LD (2014) Betaine supplementation protects against high-fructose-induced renal injury in rats.J Nutr Biochem 25:353-362.

Feig DI, Soletsky B, Johnson RJ (2008) Effect of allopurinol on blood pressure of adolescents with newly diagnosed essential hypertension: A randomized trial.J Am Med Assoc 300:924-932.

George J, Struthers AD (2009) Role of urate, xanthine oxidase and the effects of allopurinol in vascular oxidative stress. Vasc Health Risk Manag 5:265-272.

Grassi D, Ferri L, Desideri G, Di Giosia P, Cheli P, Del PR, Properzi G, Ferri C (2013) Chronic hyperuricemia, uric acid deposit and cardiovascular risk. Curr Pharm Des 19:2432-2438.

Karin M, Gallagher E (2009) TNFR signaling: Ubiquitin-conjugated TRAFfic signals control stop-and-go for MAPK signaling complexes. Immunol Rev 228:225-240.

Kikuchi H, Kogure S, Arai R, Saino K, Ohkubo A, Tsuda T, Sunaga K (2017) Rosehip inhibits xanthine oxidase activity and reduces serum urate levels in a mouse model of hyperuricemia. Biomed Rep 6:539-544.

Kim JH, Song H, Ko HC, Lee JY, Jang MG, Kim SJ (2017) Anti-oxidant and anti-inflammatory properties of Clerodendrum trichotomum leaf extracts.J Life Sci 27:640-645.

Kim KH, Kim S, Jung MY, Ham IH, Whang WK (2009) Anti-inflammatory phenylpropanoid glycosides from Clerodendron trichotomum leaves. Arch Pharm Res 32:7-13.

Lee CB (1973) An Illustrated Book of Korean Plant. Hyang Mun, Seoul, Korea.

Li Z, Sheng Y, Liu C, Li K, Huang X, Huang J, Xu K (2016) Nox4 has a crucial role in uric acid-induced oxidative stress and apoptosis in renal tubular cells. Mol Med Rep 13:4343-4348.

Mohamed AAA, Jupp OJ, Anderson HM, Littlejohn AF, Vandenabeele P, MacEwan DJ (2002) Tumor necrosis factor-induced activation of c-Jun N-terminal kinase in sensitive to caspasedependent modulation while activation of mitogen-activated protein kinase(MAPK) or p38 MAPK is not. Biochem J 366:145-155.

Neeta S, Tejas P (2007) Clerodendrum and healthcare: An overview. Med Aromatic Plant Sci Biotechnol 1:142-150.

Pacher P, Nivorozhkin A, Szabo C (2006) Therapeutic effects of xanthine oxidase inhibitors: Renaissance half a century after the discovery of allopurinol. Pharmacol Rev 58:87-114. 
Quan H, Peng X, Liu S, Bo F, Yang L, Huang Z, Li H, Chen X, Di W (2011) Differentially expressed protein profile of renal tubule cell stimulated by elevated uric acid using SILAC coupled to LC-MS. Cell Physiol Biochem 27:91-98.

Ryter SW, Otterbein LE, Morse D, Choi AMK (2002) Heme oxygenase, carbon monoxide signaling pathways: Regulation and functional significance. Mol Cell Biochem 234:249-263.

Shin HS, Kang SII, Ko HC, Park DB, Kim SJ (2017) Tangeretin improves glucose uptake in a coculture of hypertrophic adipocytes and macrophages by attenuating inflammatory changes. Dev Reprod 21:93-100.

So A, Thorens B (2010) Uric acid transport and disease. J Clin Invest 120:1791-1799.

Verzola D, Ratto E, Villaggio B, Parodi EL, Pontremoli R, Garibotto G, Viazzi F (2014) Uric acid promotes apoptosis in human proximal tubule cells by oxidative stress and the activation of NADPH oxidase NOX4. PLOS ONE 9:e115210.

Wang MX, Liu YL, Yang Y, Zhang DM, Kong LD (2015) Nuciferine restores potassium oxonateinduced hyperuricemia and kidney inflammation in mice. Eur J Pharmacol 747:59-70.

Wang WX, Xiong J, Tang Y, Zhu JJ, Li M, Zhao Y, Yang GX, Xia G, Hu JF (2013) Rearranged abietane diterpenoids from the roots of Clerodendrum trichotomum and their cytotoxicities against human tumor cells. Phytochem 89:89-95.

Wu XW, Muzny DM, Lee CC, Caskey CT (1992) Two independent mutational events in the loss of urate oxidase during hominoid evolution. J Mol Evol 34:78-84.

Zhu JX, Wang Y, Kong LD, Yang C, Zhang X (2004) Effects of Biota orientalis extract and its flavonoid constituents, quercetin and rutin on serum uric acid levels in oxonate-induced mice and xanthine dehydrogenase and xanthine oxidase activities in mouse liver.J Ethnopharmacol 93:133-140. 